\title{
An accelerated jet in DA193?
}

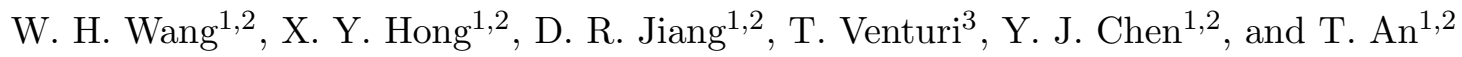 \\ 1 Shanghai Astronomical Observatory, Chinese Academy of Sciences, Shanghai 200030, PR China \\ 2 National Astronomical Observatories, Chinese Academy of Sciences, Beijing 100012, PR China \\ ${ }^{3}$ Institutor di Radioastronomia del CNR, Via P. Gobetti 101, 40129 Bologna, Italy \\ Received 14 August 2001 / Accepted 10 October 2001
}

\begin{abstract}
We present four epoch images with milliarcsecond resolution, from VLBI (Very Long Baseline Interferometry) observations of the quasar DA193 (J0555+3948) at 5GHz. The model-fitting results show that

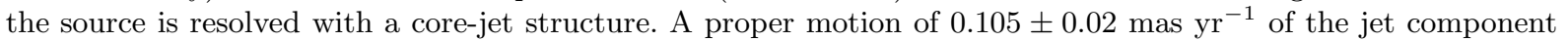
was estimated, which corresponds to an apparent velocity of $4.5 \pm 0.86 h^{-1} \mathrm{c}$ at its redshift of $z=2.365$. Estimates of the Doppler factor and of the brightness temperature confirm that this source is considerably boosted towards the line of sight. The velocity is about 4 times larger than previous estimates, which may indicate (1) that the jet component is being accelerated, possibly due to a bending jet aligned close to the line of sight; or (2) that there are two different components ejected in two flares. The possibility that multiple ejection of short-lived components is taking place in the core region of DA193 is also presented. Throughout this paper, the values $q_{0}=0.5$, $H_{0}=100 \mathrm{~km} \mathrm{~s}^{-1} \mathrm{Mpc}^{-1}$ will be used.
\end{abstract}

Key words. galaxies: jets - galaxies: quasars: general - galaxies: quasars: individual: DA193

\section{Introduction}

The study of compact radio sources with Very Long Baseline Interferometry (VLBI) has revealed some intrinsic properties of active galactic nuclei (AGN) (Pearson 1989; Cohen \& Kellermann 1995; Hirabayashi et al. 2000). The phenomenon of apparent superluminal motion, predicted by theory and found by the VLBI observations, has been explained in terms of relativistic bulk motion along (or very close to) the line of sight of the emitting plasma in AGN (Blandford 1987; Pearson \& Zensus 1987).

DA193 $\left(\mathrm{RA}_{J 2000}=05^{\mathrm{h}} 55^{\mathrm{m}} 30.806^{\mathrm{s}}, \quad \mathrm{DEC}_{J 2000}=\right.$ $\left.39^{\circ} 48^{\prime} 49.16^{\prime \prime}\right)$ is identified with an 18th magnitude lowpolarization quasar at $z=2.365$ (Wills \& Wills 1976). It is one of the most compact radio sources known to date, both on the arcsecond (Dallacasa et al. 1995; Stanghellini et al. 1990) and milliarcsecond (see for instance Fey et al. 1996) scale, and is characterised by a classical homogeneous synchrotron self-absorption spectrum, with a turnover at $5 \mathrm{GHz}$ (Spangler et al. 1983). For this reason it was classified as a GHz-peaked spectrum source (GPS) by O'Dea et al. (1991). Because of its strong and compact nature, DA193 has been observed with VLBI since the early 1980s at several frequencies. At the resolution of the order of the milliarcsecond (typical of global VLBI observations at frequencies $\nu \gtrsim 5 \mathrm{GHz}$ ) the source is only slightly resolved, and can be interpreted as the sum of a strong dominant compact component and a weaker one in PA ranging

Send offprint requests to: W. H. Wang,

e-mail: whwang@center.shao.ac.cn from $-70^{\circ}$ to $-80^{\circ}$ depending on the frequency and on the array (see for example Spangler et al. 1983, $4.99 \mathrm{GHz}$ observations carried out in August 1981; Charlot 1990, $2.3 \mathrm{GHz}$ and $8.4 \mathrm{GHz}$ observations carried out in May 1985; multiepoch observations at $2.3 \mathrm{GHz}$ and $8.4 \mathrm{GHz}$ in Fey et al. 1996); multiepoch observations at $5 \mathrm{GHz}$ in Hong et al. (1999).

On the basis of $8 \mathrm{GHz}$ multiepoch observations (1981.5, 1985.37, and 1994.9), Bajkova et al. (1996) proposed a proper motion of $0.03 \pm 0.01 \mathrm{masyr}^{-1}$. This is in agreement with an analysis carried out at $5 \mathrm{GHz}$ by Hong et al. (1999), and with more recent $43 \mathrm{GHz}$ VLBA imaging presented in Lister et al. (1998), whose submilliarcsecond scale resolution revealed a more complex structure than previously observed. In particular, beyond the extension in $\mathrm{PA} \sim-80^{\circ}$ with respect to the most compact component, another feature is visible, in $\mathrm{PA} \sim+90^{\circ}$. It is not yet clear if this component is a counterjet or the true core.

DA193 is one of the most common calibrator sources used in astronomic and geodetic VLBI observations, therefore a thorough knowledge of its radio morphology and variations is essential for its proper use as calibrator. A wealth of data in a wide range of radio wavelengths and resolutions is available for this source, and this allows us to carry out this crucial investigation.

In this paper we present four epoch VLBI images at $5 \mathrm{GHz}$, from 1996 to 1997, and estimate the proper motion of the jet components based on model-fitting results. The physical parameters of DA193, such as the equipartition Doppler factor, $\delta_{\mathrm{eq}}$, the Lorentz factor $\gamma$, the brightness 
Table 1. Telescopes used at 4 epochs and their characteristics.

\begin{tabular}{|c|c|c|c|c|c|c|c|c|}
\hline \multirow[t]{2}{*}{ No } & \multirow[t]{2}{*}{ Station } & \multirow[t]{2}{*}{ Country } & \multicolumn{4}{|c|}{ Epochs } & \multirow[t]{2}{*}{ Diameter(m) } & \multirow[t]{2}{*}{$\mathrm{SEFD}^{a}(\mathrm{Jy})$} \\
\hline & & & 96.46 & 97.13 & 97.43 & 97.85 & & \\
\hline 1 & Shanghai & China & $\sqrt{ }$ & $\sqrt{ }$ & $\sqrt{ }$ & $\sqrt{ }$ & 25 & 520 \\
\hline 2 & Nanshan & China & $\sqrt{ }$ & & & $\sqrt{ }$ & 25 & 353 \\
\hline 3 & Cambrg32M & England & $\sqrt{ }$ & & & & 32 & 136 \\
\hline 4 & Crimea & Ukrain & $\sqrt{ }$ & & $\sqrt{ }$ & & 22 & \\
\hline 5 & Eflsberg & Germany & & $\sqrt{ }$ & & $\sqrt{ }$ & 100 & 20 \\
\hline 6 & Hartrao & S. Africa & $\sqrt{ }$ & $\sqrt{ }$ & $\sqrt{ }$ & $\sqrt{ }$ & 26 & 700 \\
\hline 7 & Jodrell2 & UK & & & $\sqrt{ }$ & $\sqrt{ }$ & 25 & 320 \\
\hline 8 & Medicina & Italy & & $\sqrt{ }$ & $\sqrt{ }$ & $\sqrt{ }$ & 32 & 296 \\
\hline 9 & Noto & Italy & $\sqrt{ }$ & & $\sqrt{ }$ & $\sqrt{ }$ & 32 & 260 \\
\hline 10 & Onsala & Sweden & $\sqrt{ }$ & $\sqrt{ }$ & & & 25 & 600 \\
\hline 11 & Torun & Poland & $\sqrt{ }$ & $\sqrt{ }$ & $\sqrt{ }$ & $\sqrt{ }$ & 32 & 250 \\
\hline 12 & WSRT & Netherlands & $\sqrt{ }$ & $\sqrt{ }$ & & $\sqrt{ }$ & $92^{b}$ & 60 \\
\hline
\end{tabular}

${ }^{a}$ System equivalent flux density.

${ }^{b}$ An equivalent diameter is given.

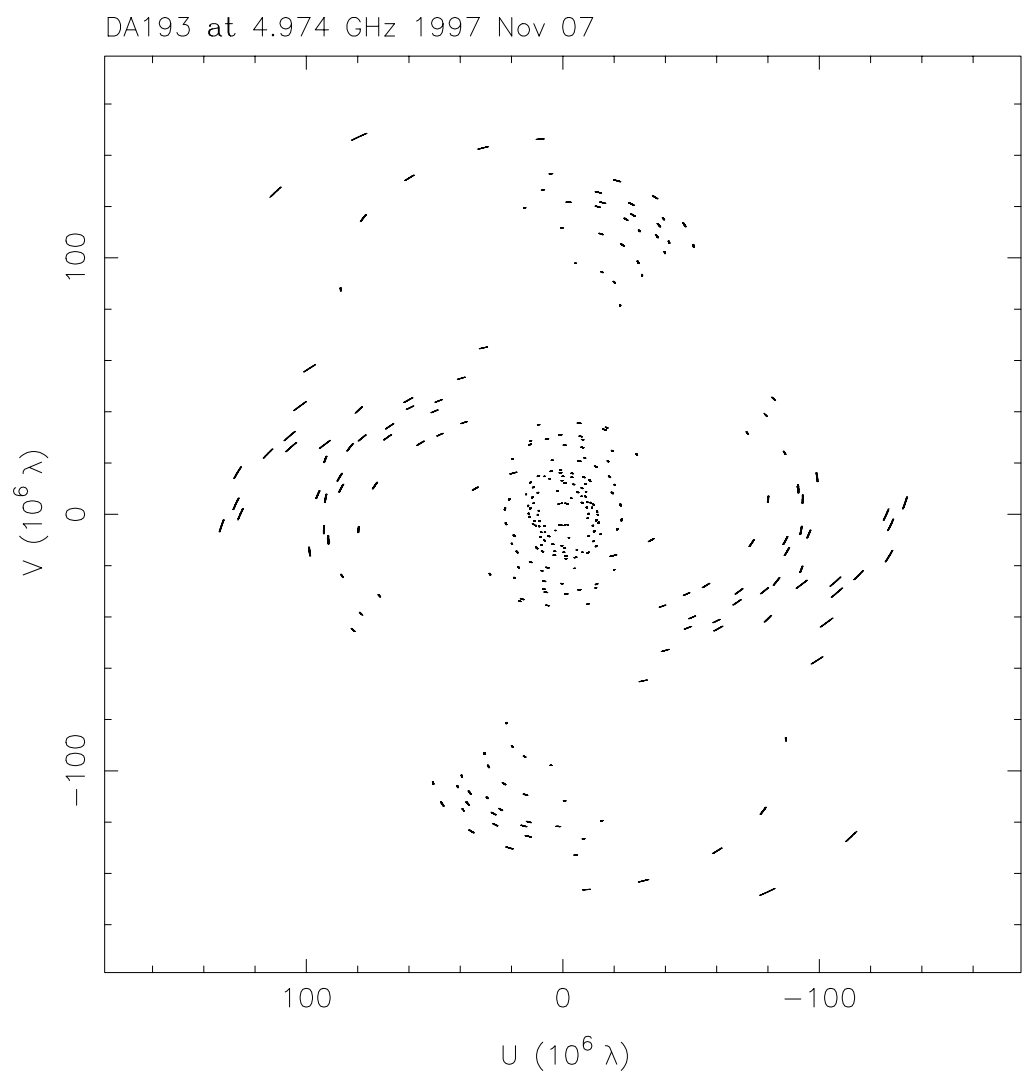

Fig. 1. The $(u, v)$ coverage of DA193 at epoch 1997.9.

temperature, $T_{\mathrm{B}}$ and the angle to the line of sight $\varphi$ are also estimated.

\section{Observations and data processing}

$5 \mathrm{GHz}$ VLBI observations of radio loud blazars, in a search for superluminal motion, were carried out with the European VLBI Network (EVN) on 17 June 1996 (Zhou et al. 2000), 18 February 1997 (Chen \& Zhang 1999),
7 June 1997 and 7 November 1997 (Hong et al. 2001) in snapshot mode. DA193 was used as a calibrator in all four experiments, and it was observed about $4-6$ times, in scans of $13 \mathrm{~min}$ each. The resolution is typically $\sim 1$ mas and the rms $\sim 7 \mathrm{mJy}^{-1}$ in the observations. The antennas involved in each epoch and their characteristics are listed in Table 1. An example of $u-v$ coverage (epoch 1997.9) is given in Fig. 1. 


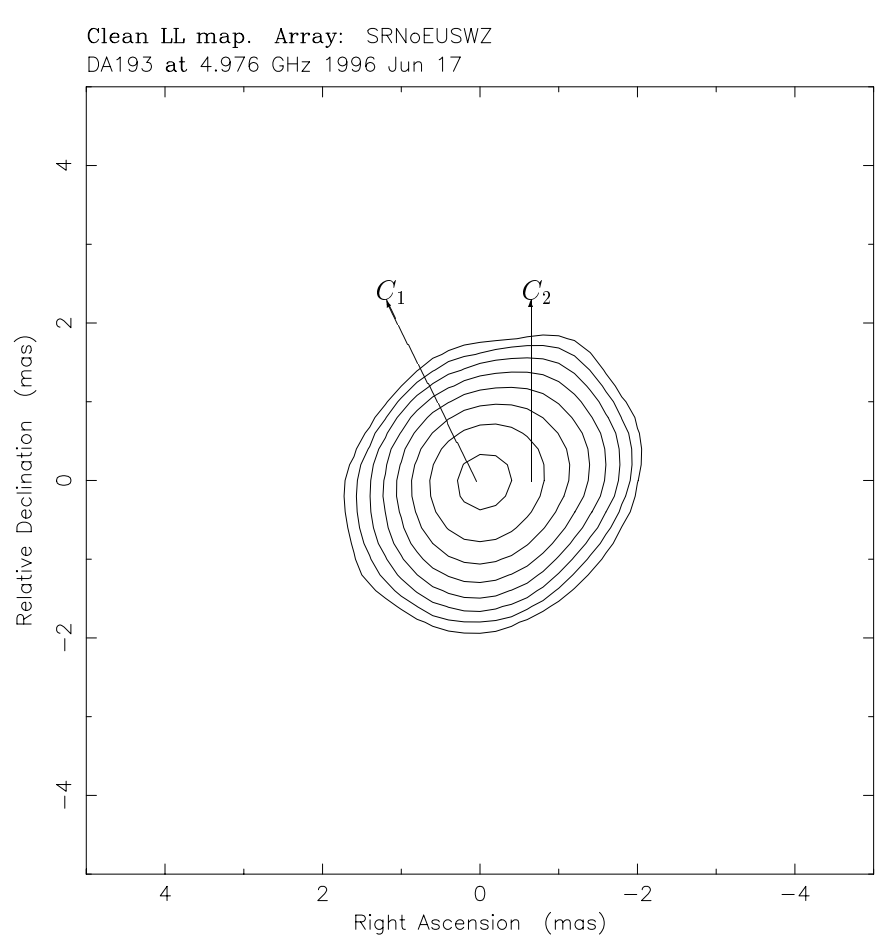

Fig. 2. The VLBI image of DA193 at $5 \mathrm{GHz}$ at Jun. 1996 Image peak: $3.2 \mathrm{Jy} \mathrm{b}^{-1}$. Contours: $0.02 \mathrm{Jy}$ $\mathrm{b}^{-1} \times(-1,1,2,4,8,16,32,64,128)$. Beam FWHM: $1.11 \times$ 0.945 (mas) at $-15.6^{\circ}$. rms: $6 \mathrm{mJy} \mathrm{b}^{-1}$.

All the data were obtained with the MK III mode B recording system (28 $\mathrm{MHz}$ bandwidth). The correlation was performed in Bonn with the Max-Planck-Institute für Radioastronomie MK III correlator with a $4 \mathrm{~s}$ integration time.

Data post-processing, including editing and amplitude calibration, were performed using the NRAO AIPS package (Cotton 1995; Diamond 1995). The hybrid images were produced by means of the package DIFMAP (Shepherd et al. 1994). A point-source model was used as an initial step for iterative self-calibration procedure for all epochs. The source was initially cleaned and phase self-calibrated using uniform weighting in the $(u, v)$ plane, then we switched to natural weighting to decrease the noise level in the image plane. The VLBI images obtained at the various epochs are displayed in Figs. 2 to 5.

\section{Analysis of the images}

As clear from the images displayed, the source is slightly resolved by our observations at all epochs.

By superresolving our images with the DIFMAP package, we have indication that it is a core-jet structure consisting of two components aligned in $\mathrm{PA}=-90^{\circ}$. The two components are labelled C1, C2 (see Figs. 2 to 5). This is consistent with the previous images and models available in the literature. We estimated the separations between the two components and their flux densities in each image by fitting a point-circle and Gaussian component model to our data using the MODELFIT program in

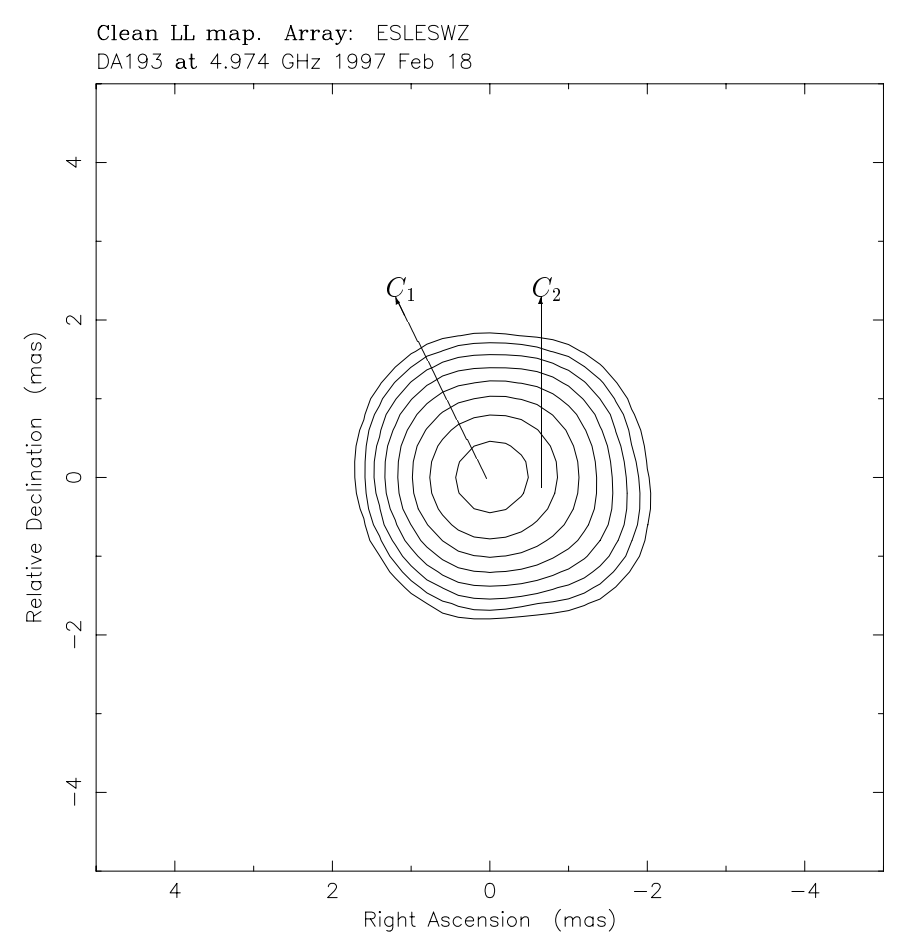

Fig. 3. The VLBI image of DA193 at 5 GHz at Feb. 1997 Image peak: $3.65 \mathrm{Jy}^{-1}$. Contours: $0.02 \mathrm{Jy}^{-1} \times$ $(-1,1,2,4,8,16,32,64,128)$. Beam FWHM: $1.18 \times 1.04$ (mas) at $-18.3^{\circ}$. rms: $7 \mathrm{mJy} \mathrm{b}^{-1}$.

the DIFMAP package. On the basis of mutil-band observations, the spectrum of $\mathrm{C} 1$ is the flattest, so we assume that $\mathrm{C} 1$ is the core of DA193.

The model-fitting results are listed in Table 2, where we also list the models available in the literature at the same frequency from 1987 to 1995.

Columns in Table 2 are as follows: Col. 1 is the observation epoch; Col. 2 is the component label; Col. 3 gives the flux density of each component; Cols. 4 and 5 list the distance and position angle of each component with respect to the strongest one; Cols. 6,7 and 8 refer to the parameters of the Gaussian model: the width at half maximum, the axis ratio and the orientation of the individual component, respectively; Col. 9 is notes. The uncertainties of the parameters are estimated with the formulae given by Fomalont (1999): $\triangle S= \pm 7 \%, \triangle r= \pm 0.05$ mas, $\triangle P A= \pm(5-10)^{\circ}, \triangle \theta_{M}= \pm 2 \%$. In Fig. 6 we plot the separation between the two components $\mathrm{C} 1$ and $\mathrm{C} 2$ vs. epoch for each epoch reported in Table 2. The core is always assumed to be stationary, so the relative proper motion of the jet can be estimated. We note that at epoch 1981.61 the jet component $\mathrm{C} 2$ is brighter than the core $\mathrm{C} 1$.

From Fig. 6 two different proper motions can be derived. The proper motion derived on the basis of the first four epochs (from 1981.61 to 1995.83), is $0.025 \pm$ $0.01 \mathrm{mas} \mathrm{yr}^{-1}$, i.e. $\beta_{\mathrm{app}}=1.1$, consistent with the result of Bajkova et al. (1996) at $8 \mathrm{GHz}$. Such a value is reported in Fig. 6 with a long-dashed line. On the basis of the five epochs from 1995 to 1997, a higher proper mo-

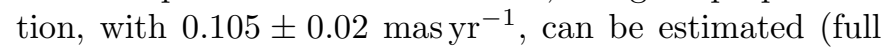




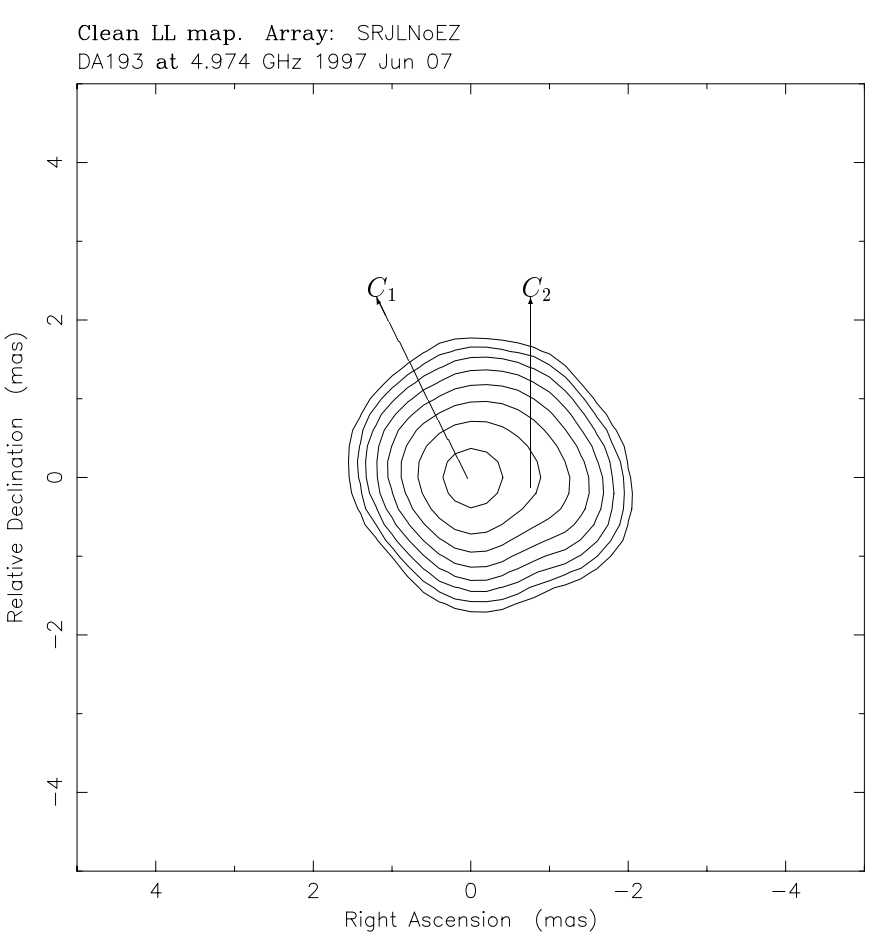

Fig. 4. The VLBI image of DA193 at $5 \mathrm{GHz}$ at Jun. 1997 Image peak: $3.45 \mathrm{Jy} \mathrm{b}^{-1}$. Contours: $0.02 \mathrm{Jy}^{-1} \times$ $(-1,1,2,4,8,16,32,64,128)$. Beam $F W H M: 1.03 \times 0.884$ (mas) at $5.65^{\circ}$. rms: $7 \mathrm{mJy} \mathrm{b}^{-1}$.

line in Fig. 6), which corresponds to an apparent velocity of $4.5 \pm 0.86 h^{-1} \mathrm{c}$.

\section{The physical parameters}

If we assume that equipartition holds in the source, it is possible to estimate the Doppler factor $\delta_{\text {eq }}$ (Readhead 1994):

$$
\begin{aligned}
\delta_{\mathrm{eq}}= & {\left[\left[10^{3} F(\alpha)\right]^{34}\left\{\left[1-(1+z)^{-1 / 2}\right] / 2 h\right\}^{-2}(1+z)^{15-2 \alpha}\right.} \\
& \left.\times S_{\mathrm{op}}^{16} \theta_{\mathrm{d}}^{-34}\left(\nu_{\mathrm{op}} \times 10^{3}\right)^{-(2 \alpha+35)}\right]^{1 /(13-2 \alpha)}
\end{aligned}
$$

where $F(\alpha)$ is given by Scott \& Readhead (1977), and $\alpha$ (assumed to be -0.75 ) is the spectral index of the radio emission. In particular, $F(\alpha)=3.4 ; z$ is the redshift, $h$ is the Hubble constant in units of $H_{0}=100 \mathrm{~km} \mathrm{~s}^{-1} \mathrm{Mpc}^{-1}$ and $S_{\text {op }}$ is the observed peak flux density in Jy at the observed frequency $\nu_{\mathrm{op}}$ in GHz. The angular diameter $\theta_{\mathrm{d}}$ (in mas) of the core component is greater than the observed angular diameter. Marscher (1987) suggested a correction for this by using $\theta_{\mathrm{d}}=1.8 \theta_{F W H M}$. We computed the equipartition Doppler factors of DA193 assuming $h=1$, $S_{\mathrm{op}}=S_{\mathrm{obs}}$, and $\nu_{\mathrm{op}}=\nu_{\mathrm{obs}}$, where $\nu_{\mathrm{obs}}$ is the frequency of our observations and $S_{\text {obs }}$ is the flux density we derived.

In the relativistic beaming model, $\beta_{\text {obs }}$ is related to the true velocity $v=\beta \mathrm{c}$, and the angle to the line of sight $\varphi$ (Pearson \& Zensus 1987),

$\beta_{\mathrm{obs}}=\frac{\beta \sin \varphi}{1-\beta \cos \varphi}$

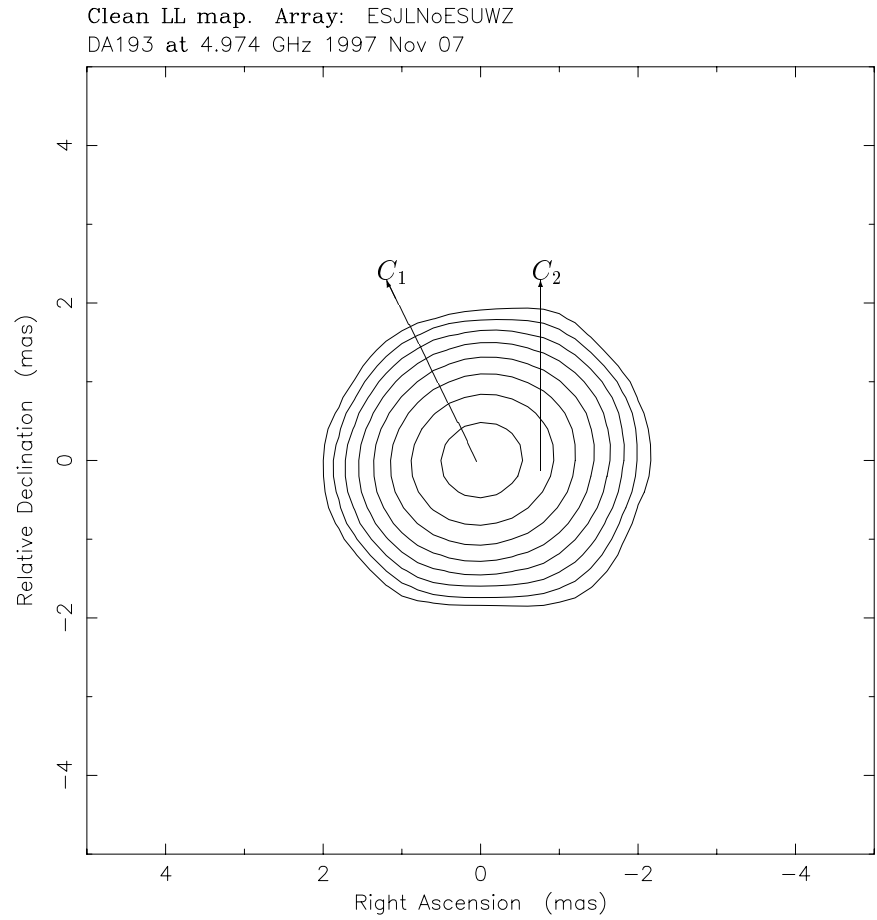

Fig. 5. The VLBI image of DA193 at $5 \mathrm{GHz}$ at Nov 1997 Image peak: $3.67 \mathrm{Jy} \mathrm{b}^{-1}$. Contours: $0.02 \mathrm{Jy}^{-1} \times$ $(-1,1,2,4,8,16,32,64,128)$. Beam $F W H M: 1.28 \times 1.16$ (mas) at $-67^{\circ}$. rms: $7 \mathrm{mJy} \mathrm{b}^{-1}$.

and the Doppler factor can be written as

$\delta=\gamma^{-1}(1-\beta \cos \varphi)^{-1}$

where $\gamma=1 / \sqrt[2]{1-\beta^{2}}$.

On the basis of the above equations, the Lorentz factor $\gamma$ and the angle to the line of sight $\varphi$ can be computed (Ghisellini et al. 1993):

$\gamma=\frac{\beta_{\mathrm{obs}}^{2}+\delta^{2}+1}{2 \delta}$

$\tan \varphi=\frac{2 \beta_{\mathrm{obs}}}{\beta_{\mathrm{obs}}^{2}+\delta^{2}-1}$

where $\beta_{\text {obs }}$ is a known superluminal velocity and $\delta$ was assumed to be $\delta=\delta_{\text {eq }}$.

The brightness temperature $T_{\mathrm{B}}$ is given (Ghisellini et al. 1993) by

$T_{\mathrm{B}}=1.77 \times 10^{12} \frac{S_{\mathrm{op}}}{\theta_{\mathrm{d}}^{2} \nu_{\mathrm{op}}^{2}}(1+z) \mathrm{K}$

where the observable quantities have the same units as in Eq. (1).

The physical parameters at each epoch are listed in Table 3. We can see that DA193 has properties of typical superluminal objects, i.e. high Doppler boosting and small viewing angle. In addition, the observed brightness temperature $T_{\mathrm{B}}$ always exceeds the inverse Compton limit (Kellermann \& Pauliny-Toth 1969). 
Table 2. VLBI component properties.

\begin{tabular}{ccccccccc}
\hline epoch & Comp & $\begin{array}{c}\mathrm{S} \\
(\mathrm{Jy})\end{array}$ & $\begin{array}{c}\mathrm{r} \\
(\mathrm{mas})\end{array}$ & $\begin{array}{c}\text { PA } \\
\left({ }^{\circ}\right)\end{array}$ & $\begin{array}{c}\theta_{\mathrm{M}} \\
(\mathrm{mas})\end{array}$ & Ratio & $\begin{array}{c}\text { pa } \\
\left({ }^{\circ}\right)\end{array}$ & Notes \\
\hline 1$)$ & $(2)$ & $(3)$ & $(4)$ & $(5)$ & $(6)$ & $(7)$ & $(8)$ & $(9)$ \\
\hline 1981.61 & $\mathrm{C} 1$ & 1.7 & & & 0.3 & 0.5 & & 1 \\
& $\mathrm{C} 2$ & 3.0 & 0.37 & & 0.88 & 1.0 & & \\
1985.37 & $\mathrm{C} 1$ & 3.0 & & & 0.73 & 0.79 & -57.87 & 2 \\
& $\mathrm{C} 2$ & 1.3 & 0.52 & -75.0 & & & & \\
1994.52 & $\mathrm{C} 1$ & 4.07 & 0.0 & 0.0 & 0.44 & 0.80 & -83 & 3 \\
& $\mathrm{C} 2$ & 1.19 & 0.70 & -74 & 0.67 & 1.0 & & \\
1995.83 & $\mathrm{C} 1$ & 4.14 & 0.0 & 0.0 & 0.62 & 0.99 & 89.20 & 4 \\
& $\mathrm{C} 2$ & 1.24 & 0.75 & -87.29 & 0.41 & 0.79 & 85.21 & \\
\hline \hline \multirow{2}{*}{1996.46} & $\mathrm{C} 1$ & 4.23 & 0.0 & 0.0 & 0.73 & 0.79 & -57.87 & 5 \\
& $\mathrm{C} 2$ & 0.98 & 0.78 & -90.56 & 1.58 & 0.46 & -22.49 & \\
1997.13 & $\mathrm{C} 1$ & 4.92 & 0.0 & 0.0 & 0.75 & 0.67 & -75.9 & 5 \\
& $\mathrm{C} 2$ & 0.53 & 0.90 & -94.3 & 2.61 & 1.0 & 41.2 & \\
1997.43 & $\mathrm{C} 1$ & 4.92 & 0.0 & 0.0 & 0.80 & 0.69 & 82.92 & 5 \\
& $\mathrm{C} 2$ & 1.33 & 0.89 & -75.25 & 1.52 & 0.40 & 42.98 & \\
1997.85 & $\mathrm{C} 1$ & 4.69 & 0.0 & 0.0 & 0.74 & 0.81 & -59.56 & 5 \\
& $\mathrm{C} 2$ & 0.32 & 0.97 & -89.01 & 1.65 & 0.27 & -8.46 & \\
\hline
\end{tabular}

Notes. - (1) Spangler et al. (1983); (2) Charlot (1990); (3) Fey et al. (1996); (4) Hong et al. (1999); (5) this paper.

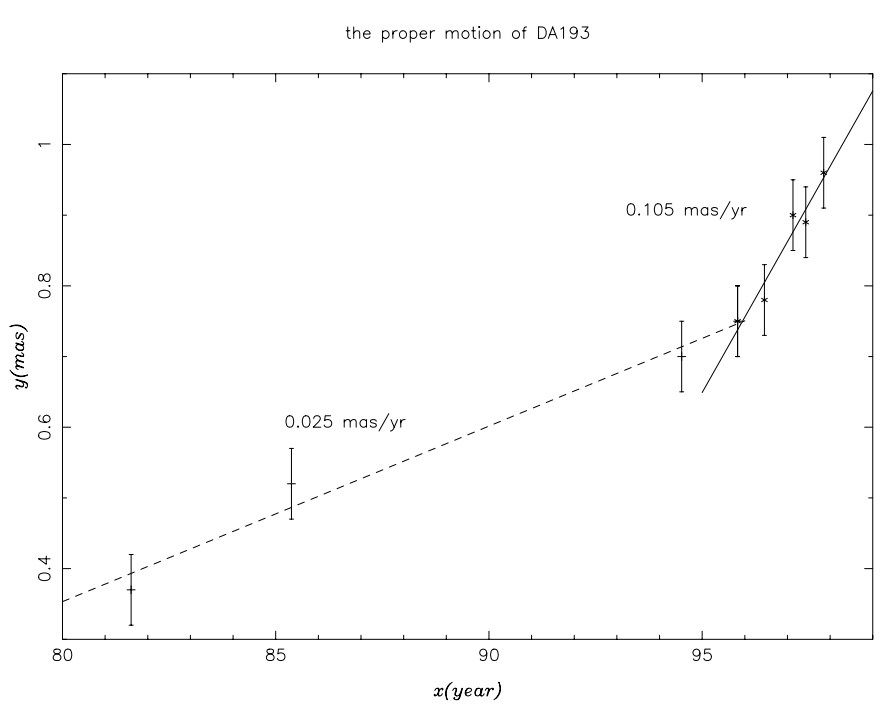

Fig. 6. Separation of two components in DA193, as function of time (from Table 2).

\section{Discussion and conclusions}

The inferred proper motion suggests DA193 as a superluminal quasar. The proper motion derived from this paper is at least 4 times larger than that estimated on the basis of the data available in the literature. Two possibilities can be taken into account:

(i) the jet component accelerates after epoch $\sim 1995$;

(ii) we track two different knots, each emitted during two different outbursts.

(i) If the jet component is the same, our results suggest that it is being accelerated. This could be due to an increase of the Lorentz factor $\gamma$, or to a change in the viewing angle, possibly associated with a change in the jet

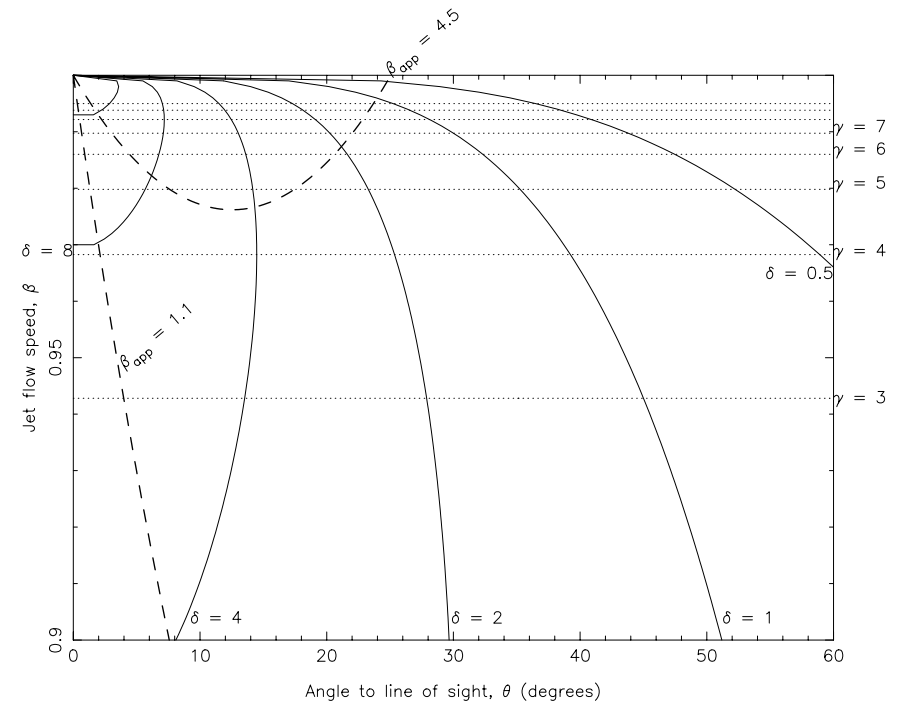

Fig. 7. Dependence of the apparent speed of the superluminal jet $\beta_{\text {app }}$ on the angle of the line of sight $\theta$ and intrinsic flow velocity $\beta$. The dashed lines show the loci on which the component is constrained by the superluminal speed, $\beta_{\text {app }}$, for 4.4 and 1.1. The horizontal dotted lines show the Lorentz factor $\gamma$ corresponding to $\beta$. The full lines are loci of constant Doppler factor $\delta$.

trajectory. The viewing angle should change by $\sim 10^{\circ}$ to explain the increase in the proper motion. Alternatively, if the viewing angle is the same at each epoch, then an intrinsic acceleration is necessary to explain the observations (see Fig. 7). We note that the proper motion we derived minimizes the Lorentz factor $\gamma$ for a viewing angle $\varphi \sim 12^{\circ}$, in reasonable agreement with the values derived in Sect. 4 on the basis of equipartition arguments.

Comparison of the previous analysis carried out by Hong et al. (1999) with the result presented in this 


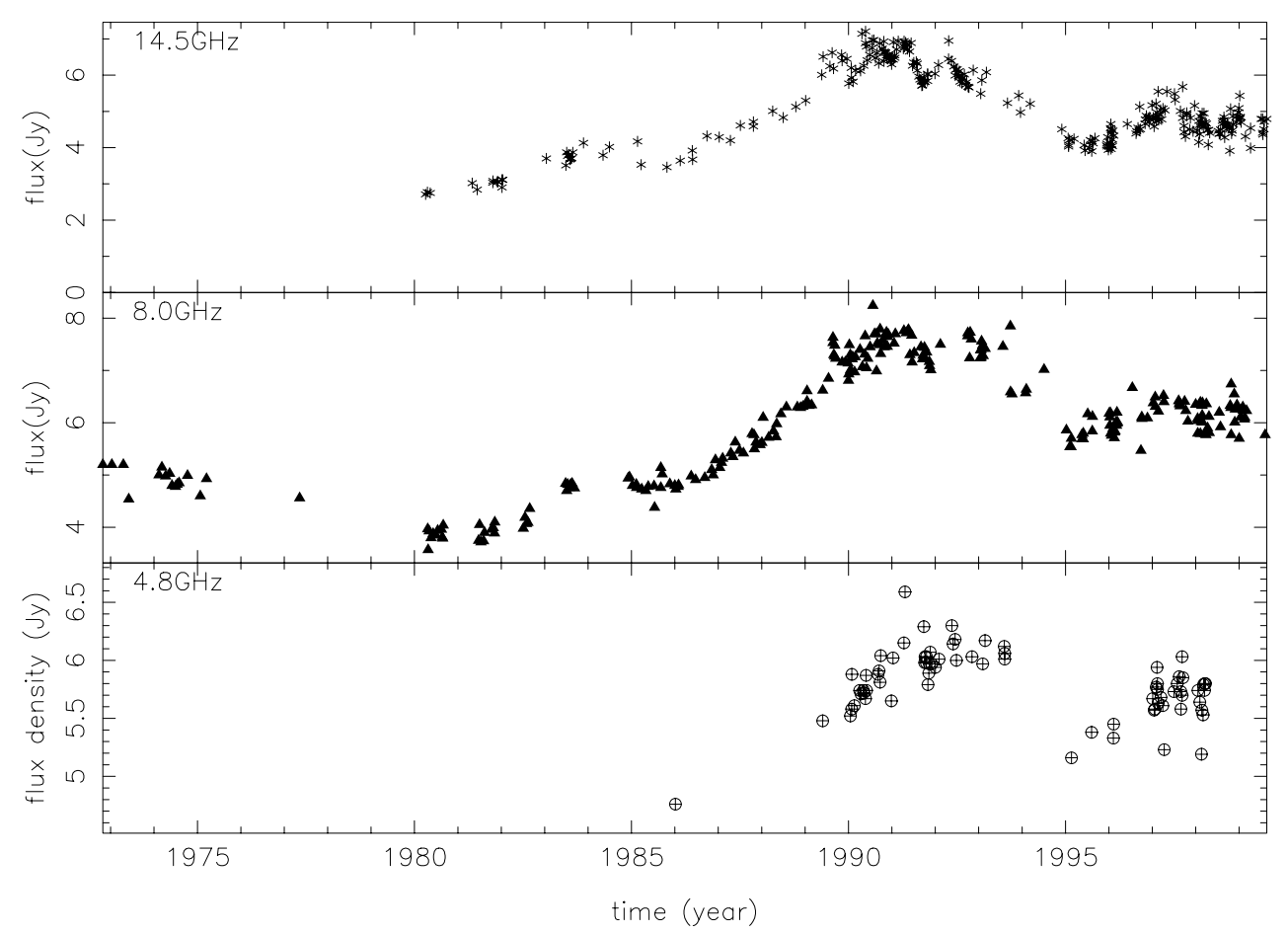

Fig. 8. The total flux densities vary with epochs at 4.8, 8.0 and $14.5 \mathrm{GHz}$ The data are archived from the University of Michigan Radio Astronomy Observatory.

paper shows that the Lorentz factor $\gamma$ did not change considerably from epoch to epoch; therefore we favour the hypothesis that the observed increase of $\beta_{\text {app }}$ is due to projection effects.

Bent jets are very common on the VLBI scales of compact radio sources, so a change of trajectory in the inner region of DA193 is not implausible. Unfortunately, the very compact nature of this source both on the arcsecond and milliarcsecond scale makes it difficult to search for other signatures of the possible bending in its morphology.

(ii) A second possibility is that we track a new component after epoch $\sim 1995$, characterised by higher apparent speed. If we extrapolate the trajectory shown in Fig. 6, we find that the birth of this component could be located between 1989 and 1990. In an attempt to find a signature of this new component in the radio flux variability, in Fig. 8 we plotted the lightcurve of DA193 from 1975 to 1999 at $4.8,8.0$ and $14.5 \mathrm{GHz}$, extracting the data from the database of the University of Michigan Radio Astronomy Observatory (UMRAO). It is clear that the flux density gradually rises from $\sim 1987$ at all frequencies, reaching a maximum first at $14.5 \mathrm{GHz}$ and $8.4 \mathrm{GHz}$ around $\sim 1990$ 1991, which propagates to $5 \mathrm{GHZ}$ with a delay of a few months. The data are suggestive of a connection between the birth of a new component and a total flux density outburst, but the evidence is not compelling.

Inspection of Table 2 and Fig. 6 suggests a third, alternative explanation. We note that component $\mathrm{C} 2$ may not
Table 3. Core physical parameters at each epoch.

\begin{tabular}{ccccc}
\hline epoch & $\gamma$ & $\delta$ & $\begin{array}{c}\varphi \\
\left(^{\circ}\right)\end{array}$ & $\begin{array}{c}T_{\mathrm{B}} \\
10^{12} \mathrm{~K}\end{array}$ \\
\hline 1996.46 & 4.94 & 6.72 & 8.01 & 1.8 \\
1997.13 & 5.62 & 8.85 & 5.29 & 2.6 \\
1997.43 & 5.0 & 6.90 & 7.71 & 2.0 \\
1997.85 & 5.12 & 7.35 & 7.03 & 2.3 \\
\hline
\end{tabular}

be the same one from epoch 1996.46 to epoch 1997.85 . The flux density of C2 decreases from $0.98 \mathrm{Jy}$ (in 1996.46) to $0.53 \mathrm{Jy}$ in 1997.13 , then it brightens again to $1.33 \mathrm{Jy}$ in the following epoch, to decrease by $\sim$ a factor of 4 in 1997.85 . Such strong variability in a jet component is difficult to explain in simple terms. One possibility is that the jet component, labelled C2 in all epochs, is very short-lived, and fades away within a few months. In this framework, component C2 in epoch 1997.43 would be a new ejection, along the same path already traced by the previous component. This possibility is supported by Fig. 6 . We note that the position of $\mathrm{C} 2$ did not change from 1997.13 to 1997.43 (see also Table 2), to speed up again in the following epoch. The separation of $\mathrm{C} 1$ and $\mathrm{C} 2$ for the four epochs presented in this paper could actually be fitted by two different lines, parallel to each other, giving support to our idea of recurrent ejection of superluminal short-lived components. It is possible to modelfit the image with three components in epoch 1997.13 because the new component 
should be visible here. Since the source DA193 is compact and the resolution is only $1.18 \times 1.04 \mathrm{mas}$, it is unlikely that three components give an equally good fitting.

Given the very compact nature of DA193, it is crucial to observe the source at submilliarcsecond resolution. To this end, either $5 \mathrm{GHz}$ Space VLBI observations or $43 \mathrm{GHz}$ ground VLBI observations are necessary tools to clarify the nature of this source, and its nuclear properties.

Acknowledgements. The authors wish to thank Dr. Daniele Dallacasa for critical reading of the manuscript and for his insightful suggestions. The authors are grateful to the staff of EVN and the Hartebeesthoek observatory for support of the observing projects. The European VLBI Network is a joint facility of European and Chinese radio astronomy institutes funded by their national research councils. The research was supported by the National Science Foundation (19973103), the Pan Deng Plan of China, and NKBRSF G19990754. This work has made use of NASA's Astrophysics Data System Abstract Service. The research has made use of data from the University of Michigan Radio Astronomy Observatory, which is supported by funds from the University of Michigan.

\section{References}

Bajkova, A. T., Pyatunina, T. B., \& Finkelstein, A. M. 1996, Commun. Inst. Appl. Astron, No. 87

Blandford, R. D. 1987, in Superluminal Radio Sources, ed. J. A. Zensus, \& T. J. Pearson (Cambridge University Press, New York), 310

Charlot, P. 1990, A\&A, 229, 51

Chen, Yong-Jun, \& Zhang, Fu-Jun 1999, Ch. A\&A, 23, 299

Cohen, M. I., \& Kellerman, K. I. (editors) 1995, Quasars and Active Galactic Nuclei: High Resolution and Radio Imaging (Publi. of the National Academy of Sciences), Conf. Proc., 92

Cotton, W. D. 1995, in Very Long Baseline Interferometry and the VLBA, ed. J. A. Zensus, P. J. Diamond, \& P. J. Napier, ASP Conf. Ser., 82, 189

Dallacasa, D., Fanti, C., Fanti, R., et al. 1995, A\&A, 295, 27

Diamond, P. J. 1995, in Very Long Baseline Interferometry and the VLBA, ed. J. A. Zensus, P. J. Diamond, \& P. J. Napier, ASP Conf. Ser., 82, 227

Fey, A. L., Clegg, A. W., \& Fomalont, E. B. 1996, ApJS, 105, 299
Fomalont, E. B. 1999, in Synthesis Imaging in Radio Astronomy II, ed. G. B. Taylor, C. L. Carilli, \& R. A. Perley, 301

Ghisellini, G., Padovani, P., Celotti, A., et al. 1993, ApJ, 407, 65

Hirabayashi, H., Pedwards, P. G., \& Murphy, D. W. (editors) 2000, Astrophycial Phenonema Revealed by Space VLBI, proceedings of the VSOP Symp., Published by the Institute of Space and Astronautical Science

Hong, X. Y., Venturi, T., Wan, T. S., et al. 1999, A\&AS, 134, 201

Hong, X. Y., Jiang, D. R., Schilizzi, R. T., et al. 2001, in Galaxies and their constituents at the highest angular resolutions, IAU Symp., 205, ed. R. T. Schilizzi, S. N. Vogel, F. Paresce, et al., ASP Conf. Ser. Publication Committee: A. V. Filipenko, G. Marcy, R. Norris, et al., 116

Kellermann, K. I., \& Pauliny-Toth, I. I. K. 1969, ApJ, 155, L71

Lister, M. L., Marscher, A. P., \& Gear, W. K. 1998, ApJ, 504, 702

Marscher, A. P. 1987, in Superluminal Radio Sources, ed. J. A. Zensus, \& T. J. Pearson (Cambridge University Press, New York), 208

O'Dea, C. P., Baum, S. A., \& Stanghellini C. 1991, ApJ, 380, 66

Pearson, T. J., \& Zensus, J. A. 1987, in Superluminal Radio Sources, ed. J. A. Zensus, \& T. J. Person (Cambridge University Press, New York), 1

Pearson, T. J. 1989, in Parsec-Scale Radio Jets, ed. J. A. Zensus, \& T. J. Person (Cambridge University Press, New York), 1

Readhead, A. C. S. 1994, ApJ, 426, 51

Shepherd, M. C., Pearson, T. J., \& Taylor, G. B. 1994, BAAS, 26, 987

Spangler, S. R., Mutel, R. L., \& Bension, J. M. 1983, ApJ, 271, 44

Stanghellini, C., Baum, S. A., Dea, C. P., et al. 1990, A\&A, 233,379

Scott, M. A., \& Readhead, A. C. S. 1977, MNRAS, 180, 539

Venturi, T., Pearson, T. J., Barthel, P. D., et al. 1993, A\&A, 271,65

Wills, D., \& Wills, B. J. 1976, ApJS, 31, 143

Zhou, J. F., Jiang, D. R., Hong, X. Y., \& Venturi, T. 2000, in Proceedings of the 5th EVN Symposium, ed. J. E. Conway, A. G. Polatidis, R. S. Booth, \& Y. M. Philström, Publ. Onsala Space Observatory, Chalmers technical University, Göteborg, Sweden, 31 